\title{
Matrix Sequences in terms of Padovan and Perrin Numbers
}

\author{
Nazmiye Yilmaz and Necati Taskara \\ Department of Mathematics, Faculty of Science, Selcuk University, Campus, 42075 Konya, Turkey
}

Correspondence should be addressed to Nazmiye Yilmaz; nzyilmaz@selcuk.edu.tr

Received 5 June 2013; Revised 29 August 2013; Accepted 13 September 2013

Academic Editor: Debasish Roy

Copyright (C) 2013 N. Yilmaz and N. Taskara. This is an open access article distributed under the Creative Commons Attribution License, which permits unrestricted use, distribution, and reproduction in any medium, provided the original work is properly cited.

The first main idea of this paper is to develop the matrix sequences that represent Padovan and Perrin numbers. Then, by taking into account matrix properties of these new matrix sequences, some behaviours of Padovan and Perrin numbers will be investigated. Moreover, some important relationships between Padovan and Perrin matrix sequences will be presented.

\section{Introduction and Preliminaries}

There are so many studies in the literature that concern the special number sequences such as Fibonacci, Lucas, Pell, Jacobsthal, Padovan, and Perrin (see, e.g., [1-4] and the references cited therein). On the other hand, the matrix sequences have taken so much interest in different types of numbers (cf. [5-7]). Therefore, a new matrix sequence related to less known numbers it is worth studying. In the light of this thought, the goal of this paper is to define the related matrix sequences for Padovan and Perrin numbers for the first time in the literature. Actually the most important difference with some other similar studies is, herein, that the study contains three-dimensional matrices instead of two as given in Fibonacci, Lucas, and Pell.

In Fibonacci numbers, there clearly exists the term Golden ratio which is defined as the ratio of two consecutive Fibonacci numbers that converges to $\alpha=(1+\sqrt{5}) / 2$. It is also clear that the ratio has so many applications in, especially, physics, engineering, architecture, and so forth $[8,9]$. In a similar manner, the ratio of two consecutive Padovan and Perrin numbers converges to

$$
\alpha_{P}=\sqrt[3]{\frac{1}{2}+\frac{1}{6} \sqrt{\frac{23}{3}}}+\sqrt[3]{\frac{1}{2}-\frac{1}{6} \sqrt{\frac{23}{3}}}
$$

that is named as Plastic constant and was firstly defined in 1924 by Gérard Cordonnier. He described applications to architecture; in 1958, he gave a lecture tour that illustrated the use of the Plastic constant in many buildings and monuments. The smallest Pisot number is the positive root of the characteristic equation $X^{3}-X-1=0$ known as the Plastic constant. This is also the characteristic equation of the recurrence equations (2) and (3), and the Plastic constant is one of its roots which is the unique real root.

Although the study of Perrin numbers started in the beginning of the 19 century under different names, the master study was published in 2006 by Shannon et al. [3]. In this reference, the authors defined the Perrin $\left\{R_{n}\right\}_{n \in \mathbb{N}}$ and Padovan $\left\{P_{n}\right\}_{n \in \mathbb{N}}$ sequences as in the forms

$$
\begin{gathered}
R_{n+3}=R_{n+1}+R_{n} \text {, where } R_{0}=3, R_{1}=0, R_{2}=2, \\
P_{n+3}=P_{n+1}+P_{n}, \text { where } P_{0}=P_{1}=P_{2}=1,
\end{gathered}
$$

respectively. It is well known that the relationship between $\left\{R_{n}\right\}_{n \in \mathbb{N}}$ and $\left\{P_{n}\right\}_{n \in \mathbb{N}}$ is presented by

$$
R_{n}=3 P_{n-5}+2 P_{n-4} .
$$

This paper is divided into two sections except the first one. In Section 2, the matrix sequences of Padovan and Perrin numbers will be defined for the first time in the literature. Then, by giving the generating functions, the Binet formulas, and summation formulas over these new matrix sequences, we will obtain some fundamental properties on Padovan and Perrin numbers. In Section 3, we will present the relationship between these matrix sequences. Since we are studying threedimensional matrices and so sequences for Padovan and Perrin numbers, there exist some difficulties in the meaning 
of the investigation of properties of Padovan and Perrin numbers. However, by the results in Sections 2 and 3 of this paper, we have a great opportunity to compare and obtain some new properties over these numbers. This is the main aim of this paper.

\section{The Matrix Sequences of Padovan and Perrin Numbers}

In this section, we will mainly focus on the matrix sequences of Padovan and Perrin numbers to get some important results. In fact, as a middle step, we will also present the related Binet formulas, summations, and generating functions. Besides, the new Binet formulas will be used in Section 3.

Hence, in the following, we will firstly define the Padovan and Perrin matrix sequences.

Definition 1. For $n \in \mathbb{N}$, the Padovan $\left(\mathscr{P}_{n}\right)$ and Perrin matrix sequences $\left(\mathscr{R}_{n}\right)$ are defined by

$$
\begin{aligned}
& \mathscr{P}_{n+3}=\mathscr{P}_{n+1}+\mathscr{P}_{n}, \\
& \mathscr{R}_{n+3}=\mathscr{R}_{n+1}+\mathscr{R}_{n},
\end{aligned}
$$

respectively, with initial conditions

$$
\begin{gathered}
\mathscr{P}_{0}=\left(\begin{array}{lll}
1 & 0 & 0 \\
0 & 1 & 0 \\
0 & 0 & 1
\end{array}\right), \quad \mathscr{P}_{1}=\left(\begin{array}{lll}
0 & 1 & 0 \\
0 & 0 & 1 \\
1 & 1 & 0
\end{array}\right), \\
\mathscr{P}_{2}=\left(\begin{array}{lll}
0 & 0 & 1 \\
1 & 1 & 0 \\
0 & 1 & 1
\end{array}\right), \\
\mathscr{R}_{0}=\left(\begin{array}{ccc}
4 & 2 & -3 \\
-3 & 1 & 2 \\
2 & -1 & 1
\end{array}\right), \quad \mathscr{R}_{1}=\left(\begin{array}{ccc}
-3 & 1 & 2 \\
2 & -1 & 1 \\
1 & 3 & -1
\end{array}\right), \\
\mathscr{R}_{2}=\left(\begin{array}{ccc}
2 & -1 & 1 \\
1 & 3 & -1 \\
-1 & 0 & 3
\end{array}\right) .
\end{gathered}
$$

In Definition 1, the matrix $\mathscr{P}_{1}$ is a matrix analogue of the Fibonacci Q-matrix which exists for Padovan numbers.

The first main result gives the $n$th general terms of the sequences in (5) and (6) via Padovan and Perrin numbers as in the following.

Theorem 2. For any integer $n \geq 0$, one has the matrix sequences

$$
\begin{aligned}
& \mathscr{P}_{n}=\left(\begin{array}{lll}
P_{n-5} & P_{n-3} & P_{n-4} \\
P_{n-4} & P_{n-2} & P_{n-3} \\
P_{n-3} & P_{n-1} & P_{n-2}
\end{array}\right), \\
& \mathscr{R}_{n}=\left(\begin{array}{lll}
R_{n-5} & R_{n-3} & R_{n-4} \\
R_{n-4} & R_{n-2} & R_{n-3} \\
R_{n-3} & R_{n-1} & R_{n-2}
\end{array}\right),
\end{aligned}
$$

respectively.
Proof. The proof will be done by induction steps.

First of all, let us consider (3) and then fix

$$
n=2, \quad n=1, \quad n=0, \quad n=-1, \quad n=-2
$$

in it. Thus we obtain the equalities $P_{-1}=P_{-3}=P_{-4}=0$ and $P_{-2}=P_{-5}=1$ which gives the following first step of the induction:

$$
\mathscr{P}_{0}=\left(\begin{array}{lll}
P_{-5} & P_{-3} & P_{-4} \\
P_{-4} & P_{-2} & P_{-3} \\
P_{-3} & P_{-1} & P_{-2}
\end{array}\right)=\left(\begin{array}{lll}
1 & 0 & 0 \\
0 & 1 & 0 \\
0 & 0 & 1
\end{array}\right) .
$$

Secondly, again considering (10) and initial condition for (3), we also get

$$
\mathscr{P}_{1}=\left(\begin{array}{lll}
0 & 1 & 0 \\
0 & 0 & 1 \\
1 & 1 & 0
\end{array}\right)
$$

Actually, by iterating this procedure and assuming the equation in (8) holds for all $n=k \in \mathbb{Z}^{+}$, we can end up the proof if we manage to show that the case also holds for $n=k+1$ :

$$
\begin{aligned}
\mathscr{P}_{k+1}= & \mathscr{P}_{k-1}+\mathscr{P}_{k-2} \\
& =\left(\begin{array}{lll}
P_{k-6} & P_{k-4} & P_{k-5} \\
P_{k-5} & P_{k-3} & P_{k-4} \\
P_{k-4} & P_{k-2} & P_{k-3}
\end{array}\right)+\left(\begin{array}{lll}
P_{k-7} & P_{k-5} & P_{k-6} \\
P_{k-6} & P_{k-4} & P_{k-5} \\
P_{k-5} & P_{k-3} & P_{k-4}
\end{array}\right) \\
& =\left(\begin{array}{ccc}
P_{k-4} & P_{k-2} & P_{k-3} \\
P_{k-3} & P_{k-1} & P_{k-2} \\
P_{k-2} & P_{k} & P_{k-1}
\end{array}\right) .
\end{aligned}
$$

Hence that is the result.

For the truthness of the Perrin matrix sequence, we need to follow almost the same approximation by considering (2). Similarly as in the above case, the final step of the induction can be obtained by $\mathscr{R}_{k+1}=\mathscr{R}_{k-1}+\mathscr{R}_{k-2}$ as follows:

$$
\mathscr{R}_{k+1}=\left(\begin{array}{ccc}
R_{k-4} & R_{k-2} & R_{k-3} \\
R_{k-3} & R_{k-1} & R_{k-2} \\
R_{k-2} & R_{k} & R_{k-1}
\end{array}\right) .
$$

This completes the proof.

Theorem 3. For every $n \in \mathbb{N}$, one can write the Binet formulas for the Padovan and Perrin matrix sequences as the form

$$
\mathscr{P}_{n}=A_{1} x^{n}+B_{1} y^{n}+C_{1} z^{n}, \quad \mathscr{R}_{n}=A_{2} x^{n}+B_{2} y^{n}+C_{2} z^{n},
$$


where

$$
\begin{gathered}
A_{1}=\frac{x \mathscr{P}_{2}+x^{2} \mathscr{P}_{1}+\mathscr{P}_{0}}{x(x-y)(x-z)}, \quad B_{1}=\frac{y \mathscr{P}_{2}+y^{2} \mathscr{P}_{1}+\mathscr{P}_{0}}{y(y-x)(y-z)}, \\
C_{1}=\frac{z \mathscr{P}_{2}+z^{2} \mathscr{P}_{1}+\mathscr{P}_{0}}{z(z-x)(z-y)}, \\
A_{2}=\frac{x \mathscr{R}_{2}+x^{2} \mathscr{R}_{1}+\mathscr{R}_{0}}{x(x-y)(x-z)}, \quad B_{2}=\frac{y \mathscr{R}_{2}+y^{2} \mathscr{R}_{1}+\mathscr{R}_{0}}{y(y-x)(y-z)}, \\
C_{2}=\frac{z \mathscr{R}_{2}+z^{2} \mathscr{R}_{1}+\mathscr{R}_{0}}{z(z-x)(z-y)},
\end{gathered}
$$

such that $x, y$, and $z$ are roots of characteristic equations of (5) and (6).

Proof. We note that the proof will be based on the recurrence relations (5) and (6) in Definition 1. As in the previous result, we will only show the truthness of the Binet formula for Padovan matrix sequence and will omit the proof of the same formula for Perrin matrix sequence since they have the same characteristic equations.

So let us consider (5). By the assumption, the roots of the characteristic equation of (5) are $x, y$, and $z$. Hence its general solution of it is given by

$$
\mathscr{P}_{n}=A_{1} x^{n}+B_{1} y^{n}+C_{1} z^{n} .
$$

Using initial conditions in Definition 1 and also applying fundamental linear algebra operations, we clearly get the matrices $A_{1}, B_{1}$, and $C_{1}$, as desired. This implies the formula for $\mathscr{P}_{n}$.

In [3], the authors obtained the Binet formulas for Padovan and Perrin numbers. Now as a different approximation and so as a consequence of Theorems 2 and 3, in the following corollary, we will present the formulas for these numbers via related matrix sequences. In fact, in the proof of this corollary, we will just compare the linear combination of the 3 rd row and 2 nd column entries of the matrices:

(i) $A_{1}, B_{1}$, and $C_{1}$ with the matrix $\mathscr{P}_{n}$ in (8) and, similarly,

(ii) $A_{2}, B_{2}$, and $C_{2}$ with the matrix $\mathscr{R}_{n}$ in (9).

Corollary 4. The Binet formulas for Padovan and Perrin numbers in terms of their matrix sequences are given by

$$
\begin{aligned}
P_{n-1}= & \frac{x^{n+3}}{(x-y)(x-z)}+\frac{y^{n+3}}{(y-x)(y-z)} \\
& +\frac{z^{n+3}}{(z-x)(z-y)}, \\
R_{n-1}= & x^{n-1}+y^{n-1}+z^{n-1},
\end{aligned}
$$

where $n>0$.
Proof. For the first part of the proof, by taking into account Definition 1 and Theorem 3, we can write

$$
\begin{aligned}
\mathscr{P}_{n}= & A_{1} x^{n}+B_{1} y^{n}+C_{1} z^{n} \\
= & \frac{x \mathscr{P}_{2}+x^{2} \mathscr{P}_{1}+\mathscr{P}_{0}}{x(x-y)(x-z)} x^{n}+\frac{y \mathscr{P}_{2}+y^{2} \mathscr{P}_{1}+\mathscr{P}_{0}}{y(y-x)(y-z)} y^{n} \\
& +\frac{z \mathscr{P}_{2}+z^{2} \mathscr{P}_{1}+\mathscr{P}_{0}}{z(z-x)(z-y)} z^{n} \\
= & \frac{x^{n-1}}{(x-y)(x-z)}\left(\begin{array}{ccc}
1 & x^{2} & x \\
x & x+1 & x^{2} \\
x^{2} & x+x^{2} & x+1
\end{array}\right) \\
& +\frac{y^{n-1}}{(y-x)(y-z)}\left(\begin{array}{ccc}
1 & y^{2} & y \\
y & y+1 & y^{2} \\
y^{2} & y+y^{2} & y+1
\end{array}\right) \\
& +\frac{z^{n-1}}{(z-x)(z-y)}\left(\begin{array}{ccc}
1 & z^{2} & z \\
z & z+1 & z^{2} \\
z^{2} & z+z^{2} & z+1
\end{array}\right) .
\end{aligned}
$$

Herein, since $x, y$, and $z$ are roots of the characteristic equation $X^{3}-X-1=0$, we clearly have

$$
\begin{aligned}
\mathscr{P}_{n}= & \frac{x^{n-1}}{(x-y)(x-z)}\left(\begin{array}{ccc}
1 & x^{2} & x \\
x & x^{3} & x^{2} \\
x^{2} & x^{4} & x^{3}
\end{array}\right) \\
& +\frac{y^{n-1}}{(y-x)(y-z)}\left(\begin{array}{ccc}
1 & y^{2} & y \\
y & y^{3} & y^{2} \\
y^{2} & y^{4} & y^{3}
\end{array}\right) \\
& +\frac{z^{n-1}}{(z-x)(z-y)}\left(\begin{array}{ccc}
1 & z^{2} & z \\
z & z^{3} & z^{2} \\
z^{2} & z^{4} & z^{3}
\end{array}\right) .
\end{aligned}
$$

Also, by Theorem 2, we obtain

$$
\begin{aligned}
\left(\begin{array}{lll}
P_{n-5} & P_{n-3} & P_{n-4} \\
P_{n-4} & P_{n-2} & P_{n-3} \\
P_{n-3} & P_{n-1} & P_{n-2}
\end{array}\right)= & \frac{x^{n-1}}{(x-y)(x-z)}\left(\begin{array}{ccc}
1 & x^{2} & x \\
x & x^{3} & x^{2} \\
x^{2} & x^{4} & x^{3}
\end{array}\right) \\
& +\frac{y^{n-1}}{(y-x)(y-z)}\left(\begin{array}{ccc}
1 & y^{2} & y \\
y & y^{3} & y^{2} \\
y^{2} & y^{4} & y^{3}
\end{array}\right) \\
& +\frac{z^{n-1}}{(z-x)(z-y)}\left(\begin{array}{ccc}
1 & z^{2} & z \\
z & z^{3} & z^{2} \\
z^{2} & z^{4} & z^{3}
\end{array}\right) .
\end{aligned}
$$

Now, if we compare the 3 rd row and 2 nd column entries with the matrices in the above equation, then we get

$$
P_{n-1}=\frac{x^{n+3}}{(x-y)(x-z)}+\frac{y^{n+3}}{(y-x)(y-z)}+\frac{z^{n+3}}{(z-x)(z-y)} .
$$


For the second part of the proof, in a similar manner, by again taking into account Theorem 3 and Definition 1, we can write

$$
\begin{aligned}
& \mathscr{R}_{n}=A_{2} x^{n}+B_{2} y^{n}+C_{2} z^{n} \\
& =\frac{x \mathscr{R}_{2}+x^{2} \mathscr{R}_{1}+\mathscr{R}_{0}}{x(x-y)(x-z)} x^{n}+\frac{y \mathscr{R}_{2}+y^{2} \mathscr{R}_{1}+\mathscr{R}_{0}}{y(y-x)(y-z)} y^{n} \\
& +\frac{z \mathscr{R}_{2}+z^{2} \mathscr{R}_{1}+\mathscr{R}_{0}}{z(z-x)(z-y)} z^{n} \\
& =\frac{x^{n-1}}{(x-y)(x-z)} \\
& \times\left(\begin{array}{ccc}
2 x-3 x^{2}+4 & -x+x^{2}+2 & x+2 x^{2}-3 \\
x+2 x^{2}-3 & 3 x-x^{2}+1 & -x+x^{2}+2 \\
-x+x^{2}+2 & 3 x^{2}-1 & 3 x-x^{2}+1
\end{array}\right) \\
& +\frac{y^{n-1}}{(y-x)(y-z)} \\
& \times\left(\begin{array}{ccc}
2 y-3 y^{2}+4 & -y+y^{2}+2 & y+2 y^{2}-3 \\
y+2 y^{2}-3 & 3 y-y^{2}+1 & -y+y^{2}+2 \\
-y+y^{2}+2 & 3 y^{2}-1 & 3 y-y^{2}+1
\end{array}\right) \\
& +\frac{z^{n-1}}{(z-x)(z-y)} \\
& \times\left(\begin{array}{ccc}
2 z-3 z^{2}+4 & -z+z^{2}+2 & z+2 z^{2}-3 \\
z+2 z^{2}-3 & 3 z-z^{2}+1 & -z+z^{2}+2 \\
-z+z^{2}+2 & 3 z^{2}-1 & 3 z-z^{2}+1
\end{array}\right) .
\end{aligned}
$$

Herein, since $x+y+z=0$ and $x y z=1$, we also clearly get

$$
\begin{aligned}
\mathscr{R}_{n}= & \frac{x^{n-1}}{2 x^{2}+(1 / x)} \\
& \times\left(\begin{array}{ccc}
2 x-3 x^{2}+4 & -x+x^{2}+2 & x+2 x^{2}-3 \\
x+2 x^{2}-3 & 3 x-x^{2}+1 & -x+x^{2}+2 \\
-x+x^{2}+2 & 3 x^{2}-1 & 3 x-x^{2}+1
\end{array}\right) \\
& +\frac{y^{n-1}}{2 y^{2}+(1 / y)} \\
& \times\left(\begin{array}{ccc}
2 y-3 y^{2}+4 & -y+y^{2}+2 & y+2 y^{2}-3 \\
y+2 y^{2}-3 & 3 y-y^{2}+1 & -y+y^{2}+2 \\
-y+y^{2}+2 & 3 y^{2}-1 & 3 y-y^{2}+1
\end{array}\right) \\
& +\frac{z^{n-1}}{2 z^{2}+(1 / z)} \\
& \times\left(\begin{array}{ccc}
2 z-3 z^{2}+4 & -z+z^{2}+2 & z+2 z^{2}-3 \\
z+2 z^{2}-3 & 3 z-z^{2}+1 & -z+z^{2}+2 \\
-z+z^{2}+2 & 3 z^{2}-1 & 3 z-z^{2}+1
\end{array}\right) .
\end{aligned}
$$

Moreover, by Theorem 2, we obtain

$$
\begin{aligned}
& \left(\begin{array}{lll}
R_{n-5} & R_{n-3} & R_{n-4} \\
R_{n-4} & R_{n-2} & R_{n-3} \\
R_{n-3} & R_{n-1} & R_{n-2}
\end{array}\right) \\
& =\frac{x^{n-1}}{2 x^{2}+(1 / x)} \\
& \quad \times\left(\begin{array}{ccc}
2 x-3 x^{2}+4 & -x+x^{2}+2 & x+2 x^{2}-3 \\
x+2 x^{2}-3 & 3 x-x^{2}+1 & -x+x^{2}+2 \\
-x+x^{2}+2 & 3 x^{2}-1 & 3 x-x^{2}+1
\end{array}\right) \\
& \quad+\frac{y^{n-1}}{2 y^{2}+(1 / y)} \\
& \quad \times\left(\begin{array}{ccc}
2 y-3 y^{2}+4 & -y+y^{2}+2 & y+2 y^{2}-3 \\
y+2 y^{2}-3 & 3 y-y^{2}+1 & -y+y^{2}+2 \\
-y+y^{2}+2 & 3 y^{2}-1 & 3 y-y^{2}+1
\end{array}\right) \\
& \quad+\frac{z^{n-1}}{2 z^{2}+(1 / z)} \\
& \quad \times\left(\begin{array}{ccc}
2 z-3 z^{2}+4 & -z+z^{2}+2 & z+2 z^{2}-3 \\
z+2 z^{2}-3 & 3 z-z^{2}+1 & -z+z^{2}+2 \\
-z+z^{2}+2 & 3 z^{2}-1 & 3 z-z^{2}+1
\end{array}\right) .
\end{aligned}
$$

Now, if we compare the 3 rd row and 2 nd column entries with the matrices in the above equation, then we get

$$
R_{n-1}=\frac{3 x^{3}-x}{2 x^{3}+1} x^{n-1}+\frac{3 y^{3}-y}{2 y^{3}+1} y^{n-1}+\frac{3 z^{3}-z}{2 z^{3}+1} z^{n-1} \text {. }
$$

Finally, since $x, y$, and $z$ are roots of the characteristic equation $X^{3}-X-1=0$, we can replace $x+1, y+1$, and $z+1$ by $x^{3}, y^{3}$, and $z^{3}$. Then we conclude that

$$
R_{n-1}=x^{n-1}+y^{n-1}+z^{n-1}
$$

as required.

Now, for Padovan and Perrin matrix sequences, we give the summations according to specified rules as we depicted at the beginning of this section.

Theorem 5. For $m>j \geq 0$, there exist

$$
\begin{aligned}
\sum_{i=0}^{n-1} \mathscr{P}_{m i+j} & \\
= & \frac{\mathscr{P}_{m n+m+j}+\mathscr{P}_{m n-m+j}+\left(1-R_{m}\right) \mathscr{P}_{m n+j}-\mathscr{P}_{m+j}}{R_{m}-R_{-m}} \\
& -\frac{\mathscr{P}_{m-j}-\left(R_{m}-1\right) \mathscr{P}_{j}}{R_{m}-R_{-m}},
\end{aligned}
$$




$$
\begin{aligned}
& \sum_{i=0}^{n-1} \mathscr{R}_{m i+j} \\
&=\frac{\mathscr{R}_{m n+m+j}+\mathscr{R}_{m n-m+j}+\left(1-R_{m}\right) \mathscr{R}_{m n+j}-\mathscr{R}_{m+j}}{R_{m}-R_{-m}} \\
&-\frac{\mathscr{R}_{m-j}-\left(R_{m}-1\right) \mathscr{R}_{j}}{R_{m}-R_{-m}} .
\end{aligned}
$$

Proof. The main point of the proof will be touched just the result Theorem 3, in other words the Binet formulas of related matrix sequences. Differently from previous results, we will consider the proof over Perrin matrix sequence and will omit the case of Padovan. Thus,

$$
\begin{aligned}
\sum_{i=0}^{n-1} \mathscr{R}_{m i+j}= & \sum_{i=0}^{n-1}\left(A_{2} x^{m i+j}+B_{2} y^{m i+j}+C_{2} z^{m i+j}\right) \\
= & A_{2} x^{j}\left(\frac{x^{m n}-1}{x^{m}-1}\right)+B_{2} y^{j}\left(\frac{y^{m n}-1}{y^{m}-1}\right) \\
& +C_{2} z^{j}\left(\frac{z^{m n}-1}{z^{m}-1}\right) .
\end{aligned}
$$

Herein, simplifying the last equality will be implied in (29) as required.

If we state almost the same explanation as in Corollary 4, then the following result will be clear for the summations of Padovan and Perrin numbers as a consequence of Theorem 5.

Corollary 6. For $m>j>0$, one has

$$
\begin{aligned}
& \sum_{i=0}^{n-1} P_{m i+j-1} \\
& =\frac{P_{m n+m+j-1}+P_{m n-m+j-1}+\left(1-R_{m}\right) P_{m n+j-1}}{R_{m}-R_{-m}} \\
& \quad-\frac{P_{m+j-1}+P_{m-j-1}-\left(R_{m}-1\right) P_{j-1}}{R_{m}-R_{-m}}, \\
& \sum_{i=0}^{n-1} R_{m i+j-1} \\
& =\frac{R_{m n+m+j-1}+R_{m n-m+j-1}+\left(1-R_{m}\right) R_{m n+j-1}}{R_{m}-R_{-m}} \\
& \quad-\frac{R_{m+j-1}+R_{m-j-1}-\left(R_{m}-1\right) R_{j-1}}{R_{m}-R_{-m}} .
\end{aligned}
$$

As we noted at the beginning of this section, the other aim of this paper is to present generating functions of our new matrix sequences. The next result deals with it.
Theorem 7. For Padovan and Perrin matrix sequences, one has the generating functions

$$
\begin{aligned}
& \sum_{i=0}^{\infty} \mathscr{P}_{i} x^{i}=\frac{1}{1-x^{2}-x^{3}}\left(\begin{array}{ccc}
1-x^{2} & x & x^{2} \\
x^{2} & 1 & x \\
x & x+x^{2} & 1
\end{array}\right) \\
& \sum_{i=0}^{\infty} \mathscr{R}_{i} x^{i} \\
& =\frac{1}{1-x^{2}-x^{3}} \\
& \quad \times\left(\begin{array}{ccc}
4-3 x-2 x^{2} & 2+x-3 x^{2} & -3+2 x+4 x^{2} \\
-3+2 x+4 x^{2} & 1-x+2 x^{2} & 2+x-3 x^{2} \\
2+x-3 x^{2} & -1+3 x+x^{2} & 1-x+2 x^{2}
\end{array}\right),
\end{aligned}
$$

respectively.

Proof. We will again omit Padovan case since the proof will be quite similar.

Assume that $G(x)$ is the generating function for the sequence $\left\{\mathscr{R}_{n}\right\}_{n \in \mathbb{N}}$. Then we have

$$
\begin{aligned}
G(x) & =\sum_{i=0}^{\infty} \mathscr{R}_{i} x^{i} \\
& =\mathscr{R}_{0}+\mathscr{R}_{1} x+\mathscr{R}_{2} x^{2}+\sum_{i=3}^{\infty}\left(\mathscr{R}_{i-2}+\mathscr{R}_{i-3}\right) x^{i} .
\end{aligned}
$$

From Definition 1, we obtain

$$
\begin{aligned}
G(x)= & \mathscr{R}_{0}+\mathscr{R}_{1} x+\mathscr{R}_{2} x^{2}-\mathscr{R}_{0} x^{2} \\
& +x^{2} \sum_{i=0}^{\infty} \mathscr{R}_{i} x^{i}+x^{3} \sum_{i=0}^{\infty} \mathscr{R}_{i} x^{i} \\
= & \mathscr{R}_{0}+\mathscr{R}_{1} x+\left(\mathscr{R}_{2}-\mathscr{R}_{0}\right) x^{2}+x^{2} G(x)+x^{3} G(x) .
\end{aligned}
$$

Now, rearrangement of the above equation will imply that

$$
G(x)=\frac{\mathscr{R}_{0}+\mathscr{R}_{1} x+\left(\mathscr{R}_{2}-\mathscr{R}_{0}\right) x^{2}}{1-x^{2}-x^{3}}
$$

which equals the $\sum_{i=0}^{\infty} \mathscr{R}_{i} x^{i}$ in the theorem.

Hence that is the result.

In [10], the authors obtained the generating functions for Padovan and Perrin numbers. However, herein, we will obtain these functions in terms of Padovan and Perrin matrix sequences as a consequence of Theorem 7 . To do that we will again compare the $3 \mathrm{rd}$ row and 2 nd column entries with the matrices in Theorem 7 . Hence we have the following corollary.

Corollary 8. There always exist

$$
\sum_{i=0}^{\infty} P_{i-1} x^{i}=\frac{x+x^{2}}{1-x^{2}-x^{3}}, \quad \sum_{i=0}^{\infty} R_{i-1} x^{i}=\frac{-1+3 x+x^{2}}{1-x^{2}-x^{3}} .
$$




\section{Relationships between New Matrix Sequences}

The following proposition (which will be needed for some of our results in this section) expresses that there always exist some interpasses between the Padovan and Perrin matrix sequences. In fact its proof can be seen directly by considering Theorem 2 and the equality in (4).

Proposition 9. For the matrix sequences $\left(\mathscr{P}_{n}\right)_{n \in \mathbb{N}}$ and $\left(\mathscr{R}_{n}\right)_{n \in \mathbb{N}}$, one has the following equalities:

(i) $\mathscr{R}_{n}=3 \mathscr{P}_{n-5}+2 \mathscr{P}_{n-4}$, for $n>4$,

(ii) $\mathscr{R}_{0} \mathscr{P}_{n}=2 \mathscr{P}_{n-2}+\mathscr{P}_{n-5}=\mathscr{R}_{n}$, for $n>4$,

(iii) $\mathscr{R}_{0} \mathscr{P}_{n}=\mathscr{P}_{n} \mathscr{R}_{0}=\mathscr{R}_{n}$.

Remark 10. We remark that the interpass between Padovan and Perrin numbers was stated in (4) as the expression of a Perrin number in terms of Padovan numbers. In addition to this, by taking into account Proposition 9, one can also obtain

$$
P_{n-1}=\frac{1}{23}\left(R_{n-3}+8 R_{n-2}+10 R_{n-1}\right)
$$

as a new interpass for the same numbers. Notice the relation in (38) based on the expression of a Padovan number in terms of Perrin numbers.

Theorem 11. For $m, n \in \mathbb{N}$, the following equalities hold:

(i) $\mathscr{P}_{m} \mathscr{P}_{n}=\mathscr{P}_{m+n}$,

(ii) $\mathscr{P}_{m} \mathscr{R}_{n}=\mathscr{R}_{n} \mathscr{P}_{m}=\mathscr{R}_{m+n}$,

(iii) $\mathscr{R}_{m} \mathscr{R}_{n}=\mathscr{R}_{n} \mathscr{R}_{m}=4 \mathscr{P}_{m+n-4}+4 \mathscr{P}_{m+n-7}+\mathscr{P}_{m+n-10}$, for $m, n>4$,

(iv) $\mathscr{R}_{m} \mathscr{R}_{n}=\mathscr{R}_{n} \mathscr{R}_{m}=2 \mathscr{R}_{m+n-2}+\mathscr{R}_{m+n-5}$, where $m>4$ or $n>4$

Proof. (i) From Theorem 3 with its assumptions, we can have

$$
\begin{aligned}
\mathscr{P}_{m} \mathscr{P}_{n}= & \left(A_{1} x^{m}+B_{1} y^{m}+C_{1} z^{m}\right) \\
& \times\left(A_{1} x^{n}+B_{1} y^{n}+C_{1} z^{n}\right) \\
= & A_{1} A_{1} x^{m+n}+A_{1} B_{1} x^{m} y^{n}+A_{1} C_{1} x^{m} z^{n} \\
& +B_{1} A_{1} y^{m} x^{n}+B_{1} B_{1} y^{m+n}+B_{1} C_{1} y^{m} z^{n} \\
& +C_{1} A_{1} z^{m} x^{n}+C_{1} B_{1} z^{m} y^{n}+C_{1} C_{1} z^{m+n} .
\end{aligned}
$$

Herein, since $x+y+z=0$ and $x y z=1$, simple matrix calculations imply that $A_{1}^{2}=A_{1}, B_{1}^{2}=B_{1}, C_{1}^{2}=C_{1}$, and

$$
A_{1} B_{1}=A_{1} C_{1}=B_{1} A_{1}=B_{1} C_{1}=C_{1} A_{1}=C_{1} B_{1}=[0] \text {. }
$$

Then we obtain

$$
\mathscr{P}_{m} \mathscr{P}_{n}=A_{1} x^{m+n}+B_{1} y^{m+n}+C_{1} z^{m+n}=\mathscr{P}_{m+n} .
$$

(ii) Here, we will just show the truthness of the equality $\mathscr{P}_{m} \mathscr{R}_{n}=\mathscr{R}_{m+n}$ since the other can be done similarly. Now, by Proposition 9(iii), we write

$$
\mathscr{P}_{m} \mathscr{R}_{n}=\mathscr{P}_{m} \mathscr{P}_{n} \mathscr{R}_{0}
$$

On the other hand, by (i) and again Proposition 9(iii), we finally have $\mathscr{P}_{m} \mathscr{R}_{n}=\mathscr{P}_{m+n} \mathscr{R}_{0}=\mathscr{R}_{m+n}$.

(iii) As in (ii), we will just show the first equality of this condition. So, by Proposition 9(ii), we have

$$
\mathscr{R}_{m} \mathscr{R}_{n}=\left(2 \mathscr{P}_{m-2}+\mathscr{P}_{m-5}\right)\left(2 \mathscr{P}_{n-2}+\mathscr{P}_{n-5}\right) \text {. }
$$

It is easy to see that one can use (i) in this latest equality. Thus, applying sufficient operations, we then obtain

$$
\mathscr{R}_{m} \mathscr{R}_{n}=4 \mathscr{P}_{n+m-4}+4 \mathscr{P}_{m+n-7}+\mathscr{P}_{m+n-10},
$$

as desired.

The final part of the proof can be seen similarly as in the proof of (iii).

Comparing matrix entries and then using Theorems 2 and 11 , we obtain the following result.

Corollary 12. One has the following identities for Padovan and Perrin numbers:

(i) $P_{m-3} P_{n-3}+P_{m-1} P_{n-2}+P_{m-2} P_{n-1}=P_{m+n-1}$,

(ii) $P_{m-3} R_{n-3}+P_{m-1} R_{n-2}+P_{m-2} R_{n-1}=R_{m+n-1}$,

(iii) $R_{m-3} R_{n-3}+R_{m-1} R_{n-2}+R_{m-2} R_{n-1}=4 P_{m+n-5}+$ $4 P_{m+n-8}+P_{m+n-11}$,

(iv) $R_{m-3} R_{n-3}+R_{m-1} R_{n-2}+R_{m-2} R_{n-1}=2 R_{m+n-3}+R_{m+n-6}$.

Proof. (i) By using Theorems 2 and 11, we have $\mathscr{P}_{m} \mathscr{P}_{n}=$ $\mathscr{P}_{m+n}$ which can be written mathematically as

$$
\begin{gathered}
\left(\begin{array}{lll}
P_{m-5} & P_{m-3} & P_{m-4} \\
P_{m-4} & P_{m-2} & P_{m-3} \\
P_{m-3} & P_{m-1} & P_{m-2}
\end{array}\right)\left(\begin{array}{lll}
P_{n-5} & P_{n-3} & P_{n-4} \\
P_{n-4} & P_{n-2} & P_{n-3} \\
P_{n-3} & P_{n-1} & P_{n-2}
\end{array}\right) \\
=\left(\begin{array}{lll}
P_{m+n-5} & P_{m+n-3} & P_{m+n-4} \\
P_{m+n-4} & P_{m+n-2} & P_{m+n-3} \\
P_{m+n-3} & P_{m+n-1} & P_{m+n-2}
\end{array}\right) .
\end{gathered}
$$

Now, by firstly multiplying the left-hand side matrices and then by comparing the 3 rd rows and 2 nd columns entries, we finally obtain the required equation in (i).

The proofs of the remaining conditions can be done similarly by considering again Theorems 11 and 2 .

Hence that is the result.

In the light of the above results, the following theorems provide us the convenience to obtain the powers of Padovan and Perrin matrix sequences.

Theorem 13. For $m, n, r \in \mathbb{N}$ and $n \geq r$, the following equalities hold:

(i) $\mathscr{P}_{n}^{m}=\mathscr{P}_{m n}$,

(ii) $\mathscr{P}_{n+1}^{m}=\mathscr{P}_{1}^{m} \mathscr{P}_{m n}$,

(iii) $\mathscr{P}_{n-r} \mathscr{P}_{n+r}=\mathscr{P}_{n}^{2}=\mathscr{P}_{2}^{n}$. 
Proof. (i) We actually can write $\mathscr{P}_{n}^{m}=\mathscr{P}_{n} \mathscr{P}_{n} \ldots \mathscr{P}_{n}(m-$ times). Now, by Theorem 11(i), we clearly obtain $\mathscr{P}_{m n}$ as the next step of this equality.

(ii) Let us consider the left-hand side of the equality. As a similar approximation in (i), we write

$$
\begin{aligned}
\mathscr{P}_{n+1}^{m} & =\mathscr{P}_{n+1} \mathscr{P}_{n+1} \cdots \mathscr{P}_{n+1} \\
& =\mathscr{P}_{m(n+1)}=\mathscr{P}_{m} \mathscr{P}_{m n}=\mathscr{P}_{1} \mathscr{P}_{m-1} \mathscr{P}_{m n} .
\end{aligned}
$$

Similarly, we can write $\mathscr{P}_{m-1}=\mathscr{P}_{1} \mathscr{P}_{m-2}$. By iterative processes, we hence obtain

$$
\mathscr{P}_{n+1}^{m}=\underbrace{\mathscr{P}_{1} \mathscr{P}_{1} \cdots \mathscr{P}_{1}}_{m \text { times }} \mathscr{P}_{m n}=\mathscr{P}_{1}^{m} \mathscr{P}_{m n} .
$$
of (ii).

The proof of (iii) can be seen quite similarly as the proof

Theorem 14. For $m, n, r \in \mathbb{N}$ and $n \geq r$, the equalities

$$
\begin{gathered}
\mathscr{R}_{n-r} \mathscr{R}_{n+r}=\mathscr{R}_{n}^{2}, \\
\mathscr{R}_{n}^{m}=\mathscr{R}_{0}^{m} \mathscr{P}_{m n}
\end{gathered}
$$

always hold.

Proof. In the first part of the proof, we mainly consider Theorem 3, in other words, the Binet formula of Perrin matrix sequence. Hence we can write

$$
\begin{aligned}
\mathscr{R}_{n-r} & \mathscr{R}_{n+r}-\mathscr{R}_{n}^{2} \\
= & \left(A_{2} x^{n-r}+B_{2} y^{n-r}+C_{2} z^{n-r}\right) \\
& \times\left(A_{2} x^{n+r}+B_{2} y^{n+r}+C_{2} z^{n+r}\right) \\
& -\left(A_{2} x^{n}+B_{2} y^{n}+C_{2} z^{n}\right)^{2},
\end{aligned}
$$

where $A_{2}, B_{2}$, and $C_{2}$ and $x, y$, and $z$ are as given in Theorem 3. By applying some elementary operations, we obtain

$$
\mathscr{R}_{n-r} \mathscr{R}_{n+r}-\mathscr{R}_{n}^{2}=D \text {, }
$$

where

$$
\begin{aligned}
D= & A_{2} B_{2} x^{n-r} y^{n-r}\left(x^{r}-y^{r}\right)^{2}+A_{2} C_{2} x^{n-r} z^{n-r}\left(x^{r}-z^{r}\right)^{2} \\
& +B_{2} C_{2} y^{n-r} z^{n-r}\left(y^{r}-z^{r}\right)^{2} .
\end{aligned}
$$

Eventually, by $A_{2} B_{2}=A_{2} C_{2}=B_{2} C_{2}=[0]_{3 \times 3}$, we get $\mathscr{R}_{n-r} \mathscr{R}_{n+r}=\mathscr{R}_{n}^{2}$, as required.

Secondly, let us consider the right-hand side of the equality $\mathscr{R}_{n}^{m}=\mathscr{R}_{0}^{m} \mathscr{P}_{m n}$. By Theorem 13(i), we have

$$
\mathscr{R}_{0}^{m} \mathscr{P}_{m n}=\underbrace{\mathscr{R}_{0} \mathscr{R}_{0} \cdots \mathscr{R}_{0}}_{m \text { times }} \underbrace{\mathscr{P}_{n} \mathscr{P}_{n} \cdots \mathscr{P}_{n}}_{m \text { times }} .
$$

By iterating usage of Proposition 9(iii), we finally obtain

$$
\begin{aligned}
\mathscr{R}_{0}^{m} \mathscr{P}_{m n} & =\mathscr{R}_{0} \mathscr{P}_{n} \mathscr{R}_{0} \mathscr{P}_{n} \cdots \mathscr{R}_{0} \mathscr{P}_{n} \\
& =\mathscr{R}_{n} \mathscr{R}_{n} \cdots \mathscr{R}_{n}=\mathscr{R}_{n}^{m} .
\end{aligned}
$$

Hence that is the result.

\section{Conflict of Interests}

We declare that there is no conflict of interests regarding the publication of this paper.

\section{Acknowledgment}

This study is a part of Nazmiye Yilmaz's Ph.D. Thesis. Thanks are due to the editor and reviewers for their interests and valuable comments.

\section{References}

[1] S. Falcón and A. Plaza, "On the Fibonacci $k$-numbers," Chaos, Solitons \& Fractals, vol. 32, no. 5, pp. 1615-1624, 2007.

[2] T. Koshy, Fibonacci and Lucas Numbers with Applications, John Wiley \& Sons, New York, NY, USA, 2001.

[3] A. G. Shannon, P. G. Anderson, and A. F. Horadam, "Properties of Cordonnier, Perrin and van der Laan numbers," International Journal of Mathematical Education in Science and Technology, vol. 37, no. 7, pp. 825-831, 2006.

[4] Y. Yazlik and N. Taskara, "A note on generalized $k$-Horadam sequence," Computers \& Mathematics with Applications, vol. 63, no. 1, pp. 36-41, 2012.

[5] H. Civciv and R. Türkmen, "On the $(s, t)$-Fibonacci and Fibonacci matrix sequences," Ars Combinatoria, vol. 87, pp. 161173, 2008.

[6] H. H. Gulec and N. Taskara, "On the $(s, t)$-Pell and $(s, t)$ Pell-Lucas sequences and their matrix representations," Applied Mathematics Letters, vol. 25, no. 10, pp. 1554-1559, 2012.

[7] Y. Yazlik, N. Taskara, K. Uslu, and N. Yilmaz, "The Generalized $(s, t)$-Sequence and its Matrix Sequence," AIP Conference Proceedings, vol. 1389, pp. 381-384, 2012.

[8] L. Marek-Crnjac, "On the mass spectrum of the elementary particles of the standard model using El Naschie's golden field theory," Chaos, Solitons and Fractals, vol. 15, no. 4, pp. 611-618, 2003.

[9] L. Marek-Crnjac, "The mass spectrum of high energy elementary particles via El Naschie's $\mathscr{E}(\infty)$ golden mean nested oscillators, the Dunkerly-Southwell eigenvalue theorems and KAM," Chaos, Solitons and Fractals, vol. 18, no. 1, pp. 125-133, 2003.

[10] A. G. Shannon and A. F. Horadam, "Generating functions for powers of third order recurrence sequences," Duke Mathematical Journal, vol. 38, pp. 791-794, 1971. 


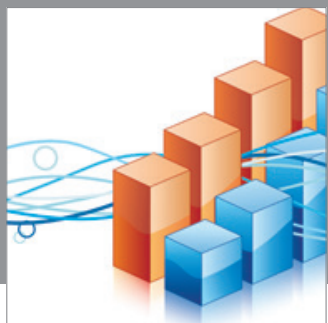

Advances in

Operations Research

mansans

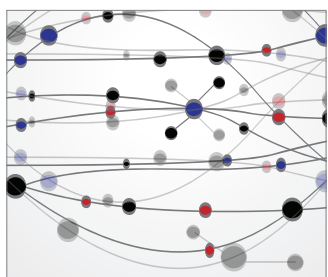

The Scientific World Journal
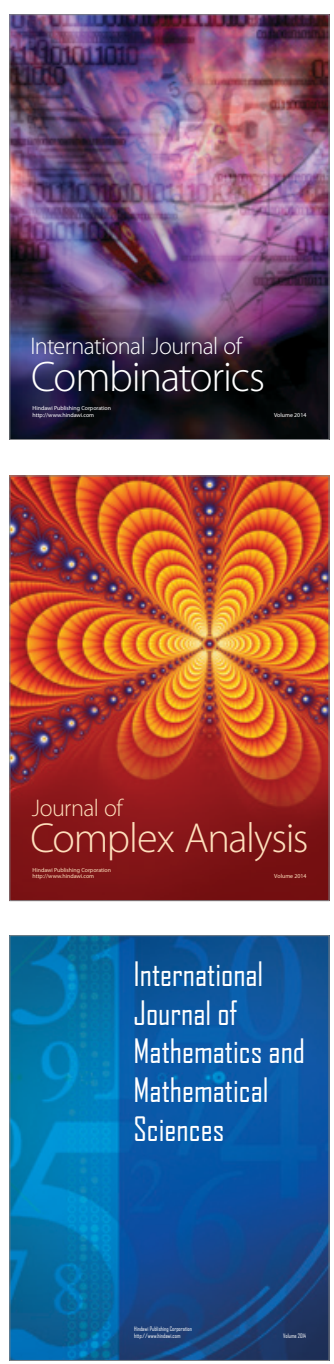
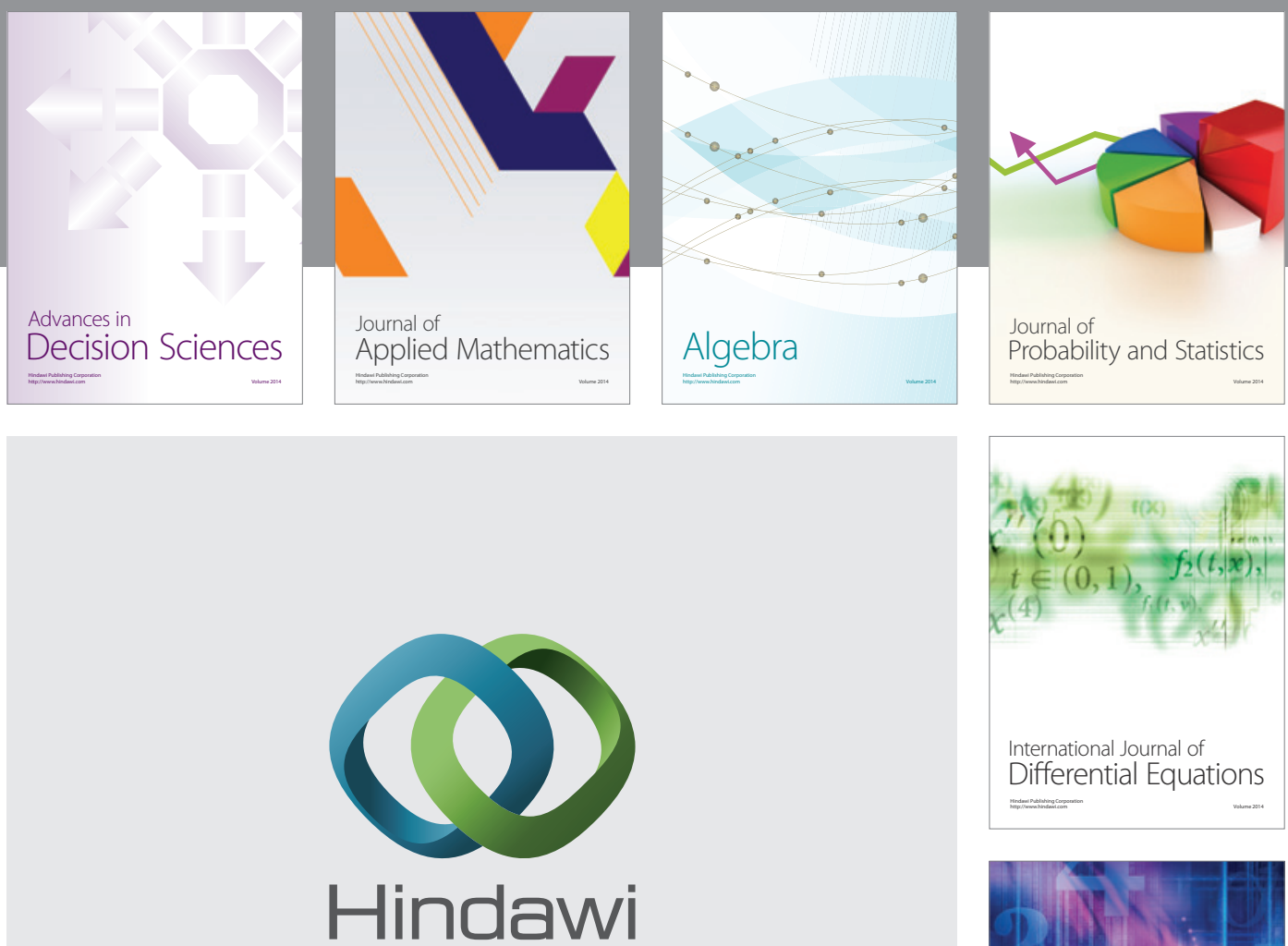

Submit your manuscripts at http://www.hindawi.com
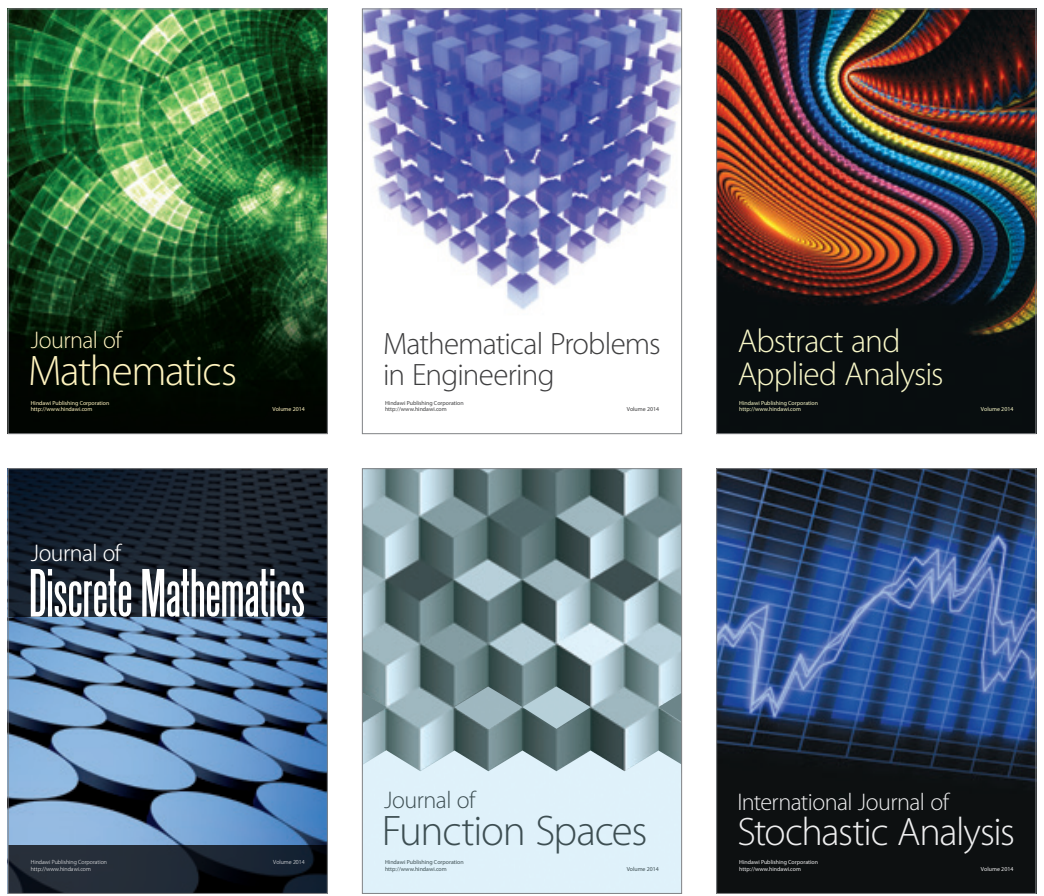

Journal of

Function Spaces

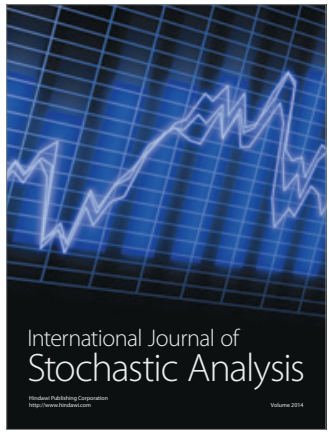

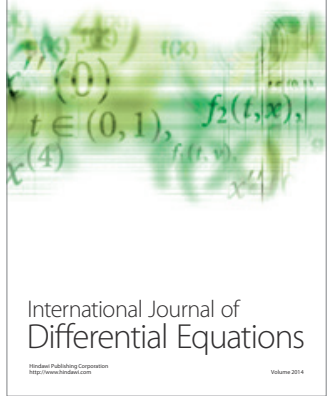
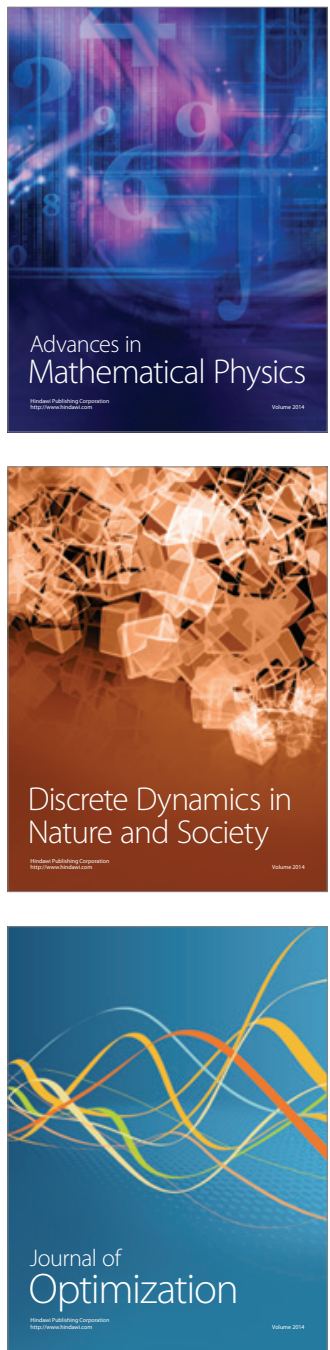